\title{
Mycobacterium bovis BCG promotes tumor cell survival from tumor necrosis factor-a-induced apoptosis
}

Sahana Holla ${ }^{\dagger}$, Devram Sampat Ghorpade ${ }^{\dagger}$, Vikas Singh, Kushagra Bansal and Kithiganahalli Narayanaswamy Balaji ${ }^{*}$

\begin{abstract}
Background: Increased incidence of lung cancer among pulmonary tuberculosis patients suggests mycobacteria-induced tumorigenic response in the host. The alveolar epithelial cells, candidate cells that form lung adenocarcinoma, constitute a niche for mycobacterial replication and infection. We thus explored the possible mechanism of M. bovis Bacillus Calmette-Guérin (BCG)-assisted tumorigenicity in type II epithelial cells, human lung adenocarcinoma A549 and other cancer cells.
\end{abstract}

Methods: Cancer cell lines originating from lung, colon, bladder, liver, breast, skin and cervix were treated with tumor necrosis factor (TNF)-a in presence or absence of BCG infection. p53, COP1 and sonic hedgehog (SHH) signaling markers were determined by immunoblotting and luciferase assays, and quantitative real time PCR was done for p53-responsive pro-apoptotic genes and SHH signaling markers. MTT assays and Annexin V staining were utilized to study apoptosis. Gain- and loss-of-function approaches were used to investigate the role for SHH and COP1 signaling during apoptosis. A549 xenografted mice were used to validate the contribution of BCG during TNF-a treatment.

Results: Here, we show that BCG inhibits TNF-a-mediated apoptosis in A549 cells via downregulation of p53 expression. Substantiating this observation, BCG rescued A549 xenografts from TNF-a-mediated tumor clearance in nude mice. Furthermore, activation of SHH signaling by BCG induced the expression of an E3 ubiquitin ligase, COP1. SHH-driven COP1 targeted p53, thereby facilitating downregulation of p53-responsive pro-apoptotic genes and inhibition of apoptosis. Similar effects of BCG could be shown for HCT116, T24, MNT-1, HepG2 and HELA cells but not for HCT116 $\mathrm{p} 53^{-/-}$and MDA-MB-231 cells.

Conclusion: Our results not only highlight possible explanations for the coexistence of pulmonary tuberculosis and lung cancer but also address probable reasons for failure of BCG immunotherapy of cancers.

Keywords: p53, TNF-a, Apoptosis, COP1, SHH signaling, BCG immunotherapy

\section{Background}

The type II alveolar epithelial cells exhibit significant functions during mycobacterial pathogenesis. Along with the alveolar macrophages, they are reservoirs for the pathogenic mycobacteria in the lungs; a site suitable for mycobacterial replication [1,2]. Additionally, these epithelial cells secrete several cytokines and chemokines that orchestrate the early host immune responses and thus establish the intra-alveolar cytokine network [3].

\footnotetext{
* Correspondence: balaji@mcbl.iisc.ernet.in

${ }^{\dagger}$ Equal contributors

Department of Microbiology and Cell Biology, Indian Institute of Science, Bangalore 560012, India
}

One of the early cytokines that could regulate epithelial responses during mycobacterial infection is tumor necrosis factor (TNF)- $\alpha$ [4]. TNF- $\alpha$ is a multifunctional cytokine that is significant for activation of macrophages and dendritic cells, granuloma formation and recruitment of lymphocytes to the infection foci during infection [5]. Of note, TNF- $\alpha$ displays potent anti-tumorigenic properties and thus used in the treatment of metastatic melanomas, primary or metastatic unresectable liver tumors and soft tissue sarcomas $[6,7]$.

In this context, a large percentage of the patients with pulmonary tuberculosis are at high risk for lung cancer, usually adenocarcinomas $[8,9]$. Many studies have linked 
carcinogenesis of the lung tissues to the genetic damage caused by infection-induced inflammation and fibrosis $[10,11]$. However, the exact cause and link between the coexistence of lung adenocarcinomas and pulmonary tuberculosis remains obscure.

One of the significant functions of the tumor suppressor p53 is to execute the apoptotic program of the cell [12]. Hence, p53 and p53-dependent regulatory network is the most common deregulated link in many human cancers including the lung cancers [13]. Transactivation properties of p53 during induction of apoptosis results in the expression of multiple apoptotic genes like $\mathrm{BH} 3-$ only family PUMA, NOXA, BID and Bcl-2 family $B A X$ [12]. Interestingly, the turnover of p53 protein is crucial to determine the cell-fate and is tightly regulated by multiple E3 ubiquitin ligases and the proteasome machinery $[14,15]$. Various cues including treatment with cytokines like TNF- $\alpha$ induces the transcriptional activation and stabilization of p53 [16].

Several signaling pathways including sonic hedgehog $(\mathrm{SHH})$ signaling, regulate the cellular homeostasis. $\mathrm{SHH}$ signaling exhibits myriad functions during embryonic development, wound healing, tissue and organ development and infections [17]. Evidently, deregulated $\mathrm{SHH}$ signaling is often associated with many human cancers [18]. Canonical SHH signaling involves its binding to the receptor, Patched-1 (PTCH1), alleviating the inhibition on Smoothened (SMO). Subsequently, SMO leads to the activation of GLI family of transcription factors (GLI1 and GLI2). While inhibitory complex comprising of GSK-3 $\beta$ is inactivated, GLI1 repressor NUMB is degraded. Thus, GLI1 is now functional to transactivate the responsive genes [19].

In the current investigation, we have explored the possible mechanism of mycobacteria-assisted tumorigenicity in type II epithelial cells, A549 human lung adenocarcinoma. Inhibition of TNF- $\alpha$-induced apoptosis was identified as the mechanism of BCG action as assessed in A549 and several other tumor cells. Moreover, we found that BCG activated the $\mathrm{SHH}$ signaling to express an E3 ubiquitin ligase, constitutively photomorphogenic 1 (COP1)/ RFWD2 which targets TNF- $\alpha$-responsive p53 and thus subdues apoptosis. Classical xenograft studies in nude mice showed that BCG suppressed the TNF- $\alpha$ action of tumor clearance; rather promoted tumor formation. Altogether, our results provide the molecular basis of inhibition of apoptosis, executed by mycobacteria in several tumors to promote tumorigenesis.

\section{Results}

\section{BCG targets p53 to rescue TNF-a-mediated cell death}

To investigate the effect of mycobacterial infection on the tumorigenic properties of alveolar epithelial cells, we performed co-treatment of A549 cells with BCG and
TNF- $\alpha$, a primary cytokine produced during infection [4]. Previous reports suggest that TNF- $\alpha$ induces $\mathrm{p} 53$ expression [16] and lung cancers are often associated with deregulated p53 [20]. We thus analyzed the expression of p53 in the current scenario. TNF- $\alpha$ induced p53 expression in A549 cells as assessed by protein levels as well as its promoter activity (Figure $1 \mathrm{~A}$ and B). Surprisingly, infection of A549 cells with BCG decreased the expression of TNF- $\alpha$-stimulated p53 (Figure $1 C$ ). p53 promoter reporter analysis confirmed the same (Figure 1D). p53 is a tumor suppressor that has been strongly implicated in the process of cell death, especially apoptosis [12]. MTT assays confirmed the ability of BCG to rescue cells from TNF- $\alpha$-stimulated cell death (Figure 1E). Of note, TNF- $\alpha$ driven apoptosis is one of the known mechanisms of tumor clearance [21]. Hence, we analyzed the expression of p53-transactivated apoptotic genes such as CDKN1A/ $p 21, N O X A$ and PUMA. In accordance with the expression of $\mathrm{p} 53$, while the responsive genes were induced upon TNF- $\alpha$ treatment, BCG suppressed TNF- $\alpha$-stimulated expression of CDKN1A, NOXA and PUMA (Figure 1F). Further, as illustrated in Figure $1 G$ and Additional file 1: Figure S1, TNF- $\alpha$ treatment induced apoptosis in lung adenocarcinoma A549 cells. However, BCG-infected cells inhibited TNF- $\alpha$-arbitrated apoptosis as assessed by Annexin V staining. Together, these results suggest that TNF- $\alpha$ mediates apoptosis via p53 action in A549 cells and BCG surmounts this process.

\section{BCG induces COP1 via SHH signaling to regulate p53-mediated apoptosis}

p53 turnover in the cells is often regulated by many E3 ubiquitin ligases including COP1 [15]. Therefore, we examined the expression of COP1 in response to BCG treatment. Notably, treatment of cells with BCG resulted in marked increase of COP1 transcripts as well as the protein (Figure 2A,B and C). Furthermore, while BCG failed to downregulate TNF- $\alpha$-induced p53 expression in cells transfected with COP1 siRNA (Figure 2D and E), enforced expression of COP1 significantly reduced the TNF- $\alpha$-responsive p53 (Figure 2F) and p53-inducible apoptotic genes (Figure 2G) in the cells. Substantiating these results, COP1 overexpression downregulated TNF- $\alpha$ (Figure 2H) or p53- (Figure 2I) stimulated apoptosis.

We then explored the molecular mechanisms that promoted BCG-driven COP1 expression in cells. Oncogenic properties of $\mathrm{SHH}$ signaling are well established and recent evidences suggested the role of $\mathrm{SHH}$ signaling in suppressing p53-mediated apoptosis [22]. Further, BCG was found to activate $\mathrm{SHH}$ signaling in macrophages to regulate immune functions [23,24]. To determine the contribution of $\mathrm{SHH}$ signaling in the current study, we first examined the activation of $\mathrm{SHH}$ signaling in A549 cells on treatment with BCG. Activation of canonical 


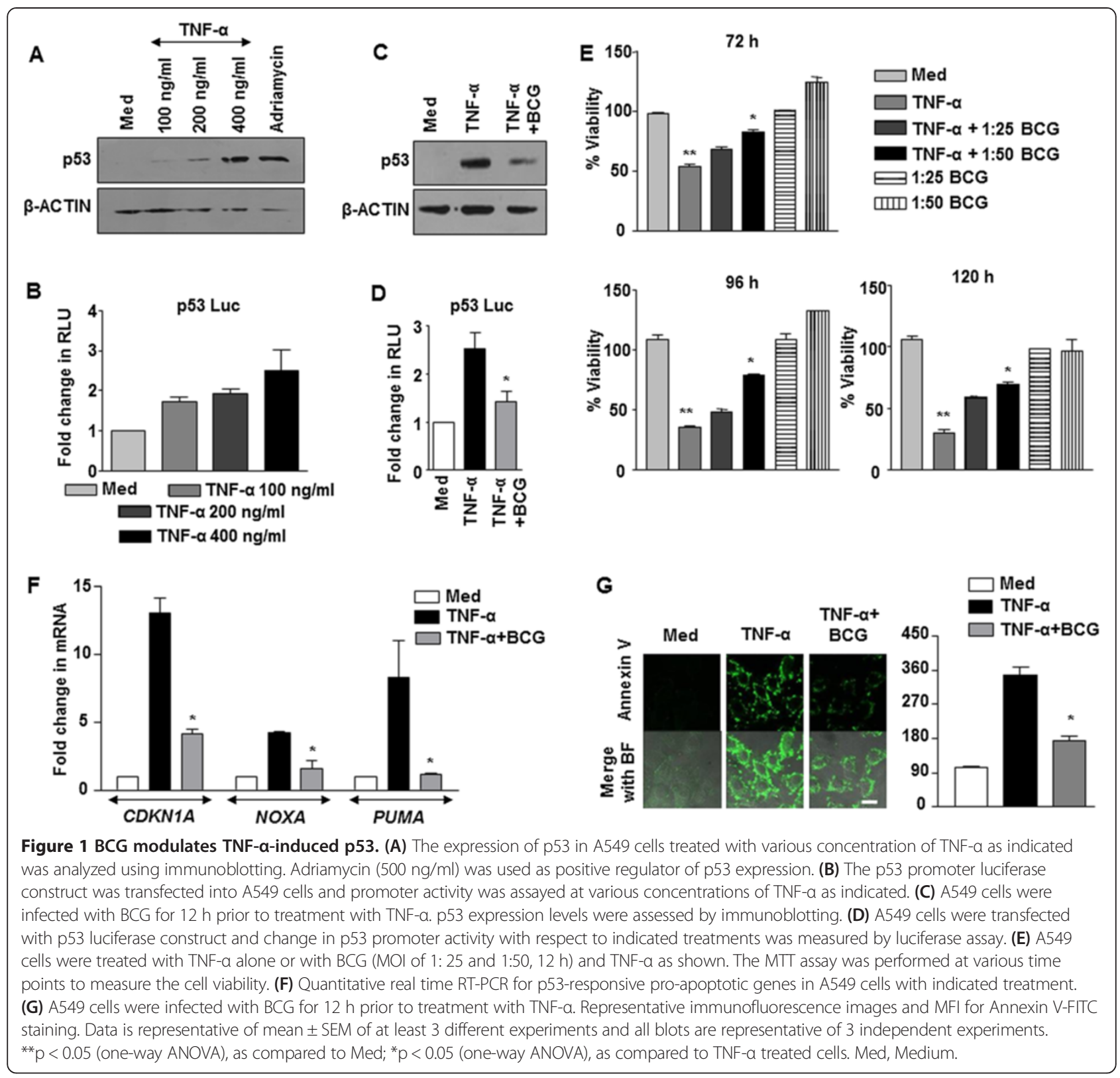

SHH signaling is assessed by transcript analysis of $S H H$, GLI1, GLI2, PTCH1, SMO and protein level expression of SHH, NUMB and GSK-3ß. Elevated transcripts, increased SHH protein, pGSK-3 $\beta$ (Ser9) and decreased NUMB indicates active $\mathrm{SHH}$ signaling. Utilizing these readouts, BCG was found to induce $\mathrm{SHH}$ signaling in A549 cells (Figure 3A and B). Additionally, inhibition of SHH signaling in A549 cells by specific pharmacological inhibitors (Figure 3C,D,E and F) or by RNA interference of $\mathrm{SHH}$ (Figure 3G,H and I) abrogated BCG-induced COP1 expression and p53 downregulation. Elevated transcripts and promoter activity of COP1 was detected on $\mathrm{SHH}$ overexpression (Figure 3J). Importantly, ChIP assay showing recruitment of GLI1 transcription factor to the COP1 promoter in response to BCG treatment indicated that $\mathrm{SHH}$ signaling could regulate COP1 expression (Figure 3K). Corroborating these observations, we found that ability of BCG to inhibit TNF- $\alpha$-stimulated apoptosis was severely compromised in cells that were either pretreated with $\mathrm{SHH}$ inhibitors (Figure 4A) or transfected with $\mathrm{SHH}$ siRNA (Figure 4B). SHH overexpression was sufficient to significantly subdue TNF- $\alpha$-mediated apoptosis (Figure 4C) and expression of apoptotic genes CDKN1A, NOXA and PUMA (Figure 4D). Further, 


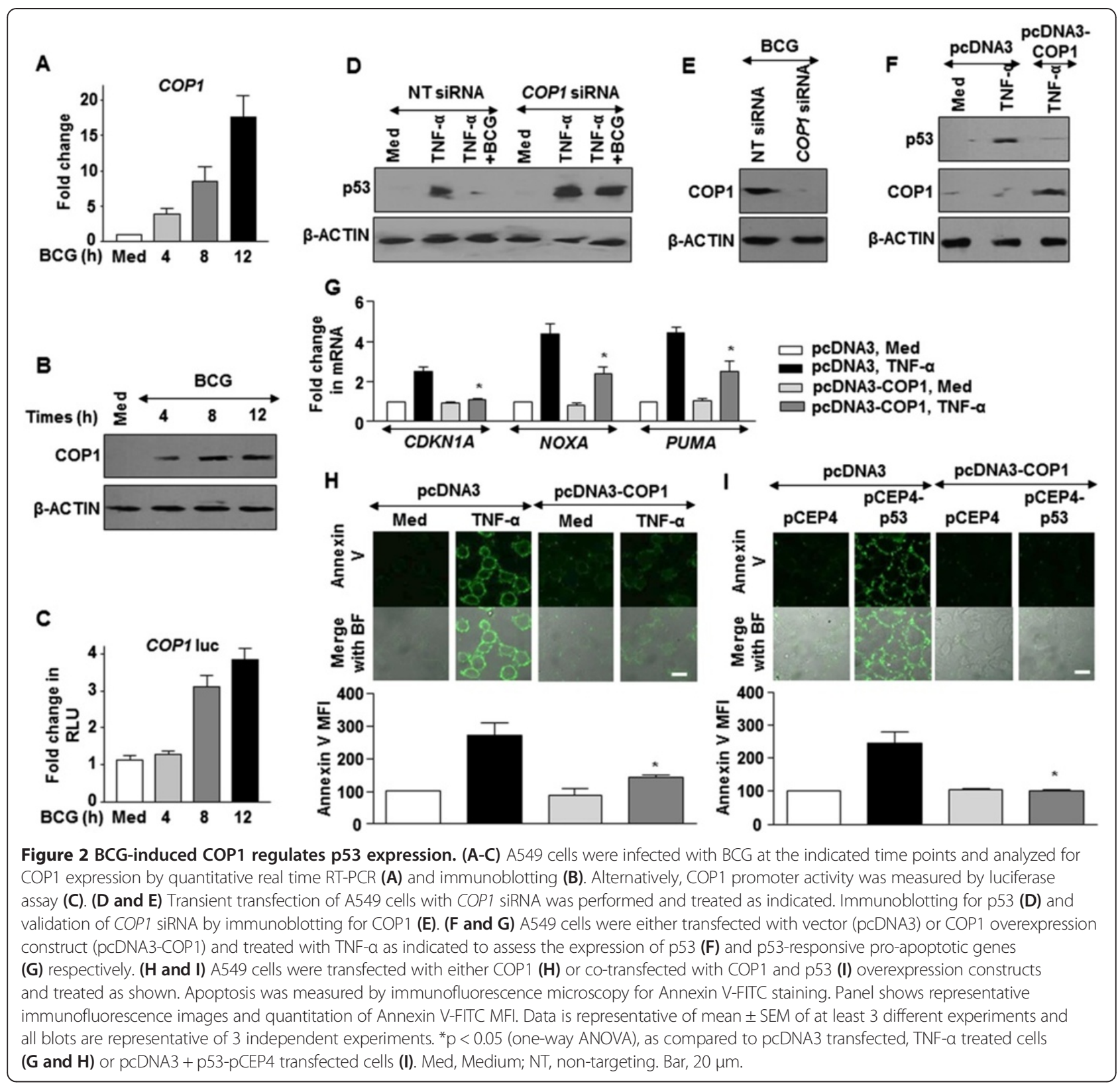

enhanced K48 ubiquitination of p53 and interaction of COP1 and p53 on BCG pretreatment of A549 cells complement the observations (Figure 4E).

\section{BCG protects tumors from TNF- $a$-mediated apoptosis}

To evaluate these observations in vivo, we chose to analyze the progression of human lung adenocarcinoma A549 xenografts on co-treatment with TNF- $\alpha$ and BCG. Ascertaining the cell line data, while TNF- $\alpha$-treated xenografts failed to exhibit sustained tumors in nude mice, cells that were infected with BCG prior to TNF- $\alpha$ treatment or infected with BCG alone formed significantly larger tumors (Figure 5A,B and C). This observation was unlike many studies that attributed anti-tumorigenic abilities of BCG instillations [25].

\section{Inhibition of TNF-a-orchestrated apoptosis by BCG in} tested cancers

BCG is utilized for intravesical immunotherapy for treating cancers such as bladder cancer [26,27], lung cancer $[28,29]$, colorectal cancer [30], melanomas [31,32] hepatocellular carcinoma [33] and cervical cancer [34]. However, large proportion of the patients fails such immunotherapies [27]. Further, TNF- $\alpha$ is also used for cancer therapy $[6,7]$. Hence, with the current results, we analyzed the effect of BCG instillation during TNF- $\alpha$ treatment of 


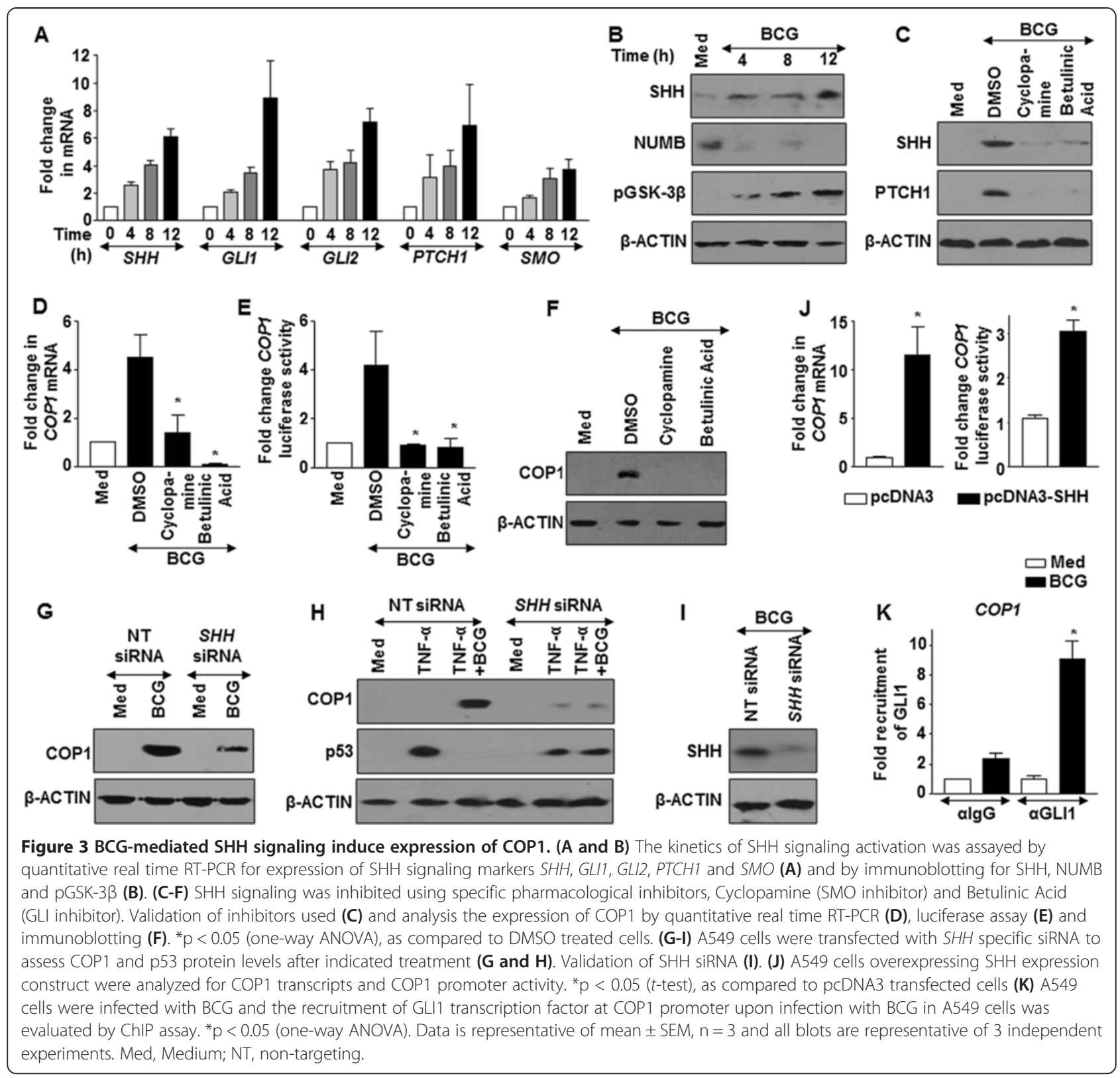

various tumor cell lines. Corresponding our previous observation, except in HCT116 $\mathrm{p} 53^{-/-}$human colorectal carcinoma cells and MDA-MB-231 human breast adenocarcinoma cells, infection of the HCT116 human colorectal carcinoma cells, T24 human urinary bladder carcinoma cells, MNT-1 human melanoma cells, HepG2 human hepatocellular carcinoma cells or HELA human cervical adenocarcinoma cells with BCG decreased the expression of TNF- $\alpha$-responsive p53 and induced the COP1 expression (Figure 5D). The expression of p53-dependent apoptotic genes such as CDKN1A, NOXA and PUMA followed similar trend (Figure 5E and F). Finally, the ability of BCG to protect the tested tumor cells from TNF- $\alpha$-mediated apoptosis was also observed in HCT116, T24, MNT-1,
HepG2 and HELA cells but not in HCT116 p53 ${ }^{-/-}$cells and MDA-MB-231 cells (Figure 6A and B). In conclusion, these results suggest that inhibition of apoptosis by BCG could fail intravesical immunotherapy.

\section{Discussion}

Interaction of the pathogenic mycobacteria with macrophages and epithelial cells in the alveolar spaces shape the early responses of the infection $[1,2,35]$. Reports suggest that apart from harboring the bacteria, infectioninduced cytokines and chemokines secreted by alveolar type II epithelial cells recruit other immune cells such as monocytes, lymphocytes and polymorphonuclear cells [3]. Of note, association of pulmonary tuberculosis with 


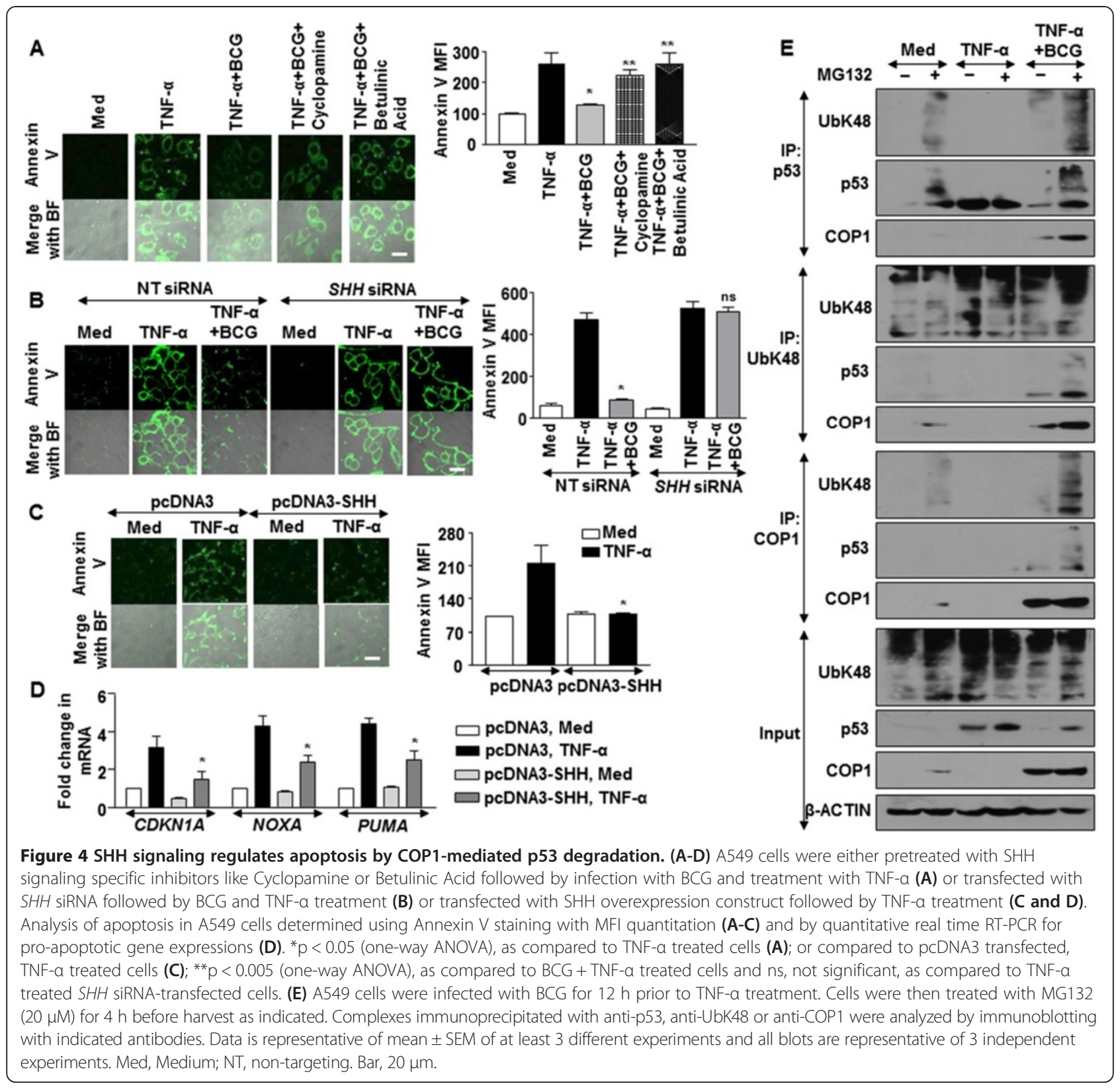

lung cancer has underscored the crucial role for the infected alveolar epithelial cells in maintaining immune and cellular homeostasis during mycobacterial infection $[8,9]$. However, the mechanism of infection-induced deregulation is not clear. Here, we found that BCG inhibits the TNF- $\alpha$-mediated induction of a tumor suppressor, p53 and thus promotes tumor growth.

p53 exhibits a diverse role as a tumor suppressor and hence is called as the guardian of the genome [36]. A number of investigations have emphasized the tumor surveillance property of p53, especially its pro-apoptotic functions [12,37]. In the current context, BCG targets p53-mediated apoptosis of the cancer cells. Further,
COP1 is a RING-finger containing E3-ubiquitin ligase which can maintain the levels of multiple cellular proteins including p53 [15]. Interestingly, reports suggest that COP1 exhibits both oncogenic and tumor suppressive functions depending on the cancer type [38]. Here, we find the oncogenic property of BCG-driven COP1 to promote tumor formation and maintenance by targeting p53.

Multiple signaling pathways are tightly regulated to assert cellular homeostasis and maintenance. Deregulation of such pathways is often implicated in several cancers [39]. Recent studies indicate that aberrant SHH signaling leads to tumorigenesis in several tissues by inducing 


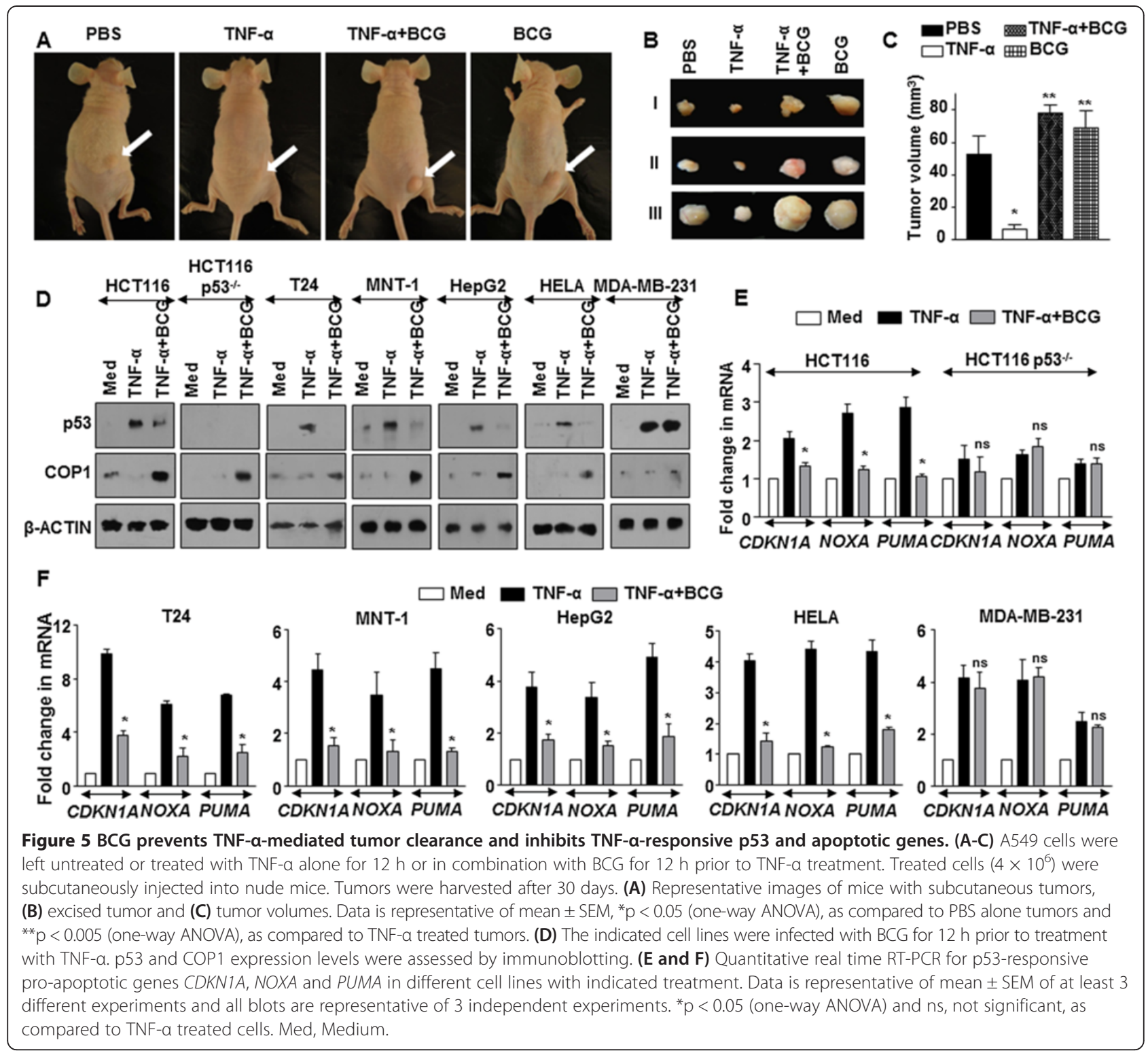

excessive proliferation and differentiation [18]. In this context, ability of BCG to activate cellular signaling pathways including $\mathrm{SHH}$ cascade is well evidenced in macrophages $[23,40,41]$. In the present study, we found that BCG could elevate SHH signaling in A549 cells. Supporting the previous observation [22], the induced $\mathrm{SHH}$ signaling inhibits p53-stimulated apoptosis. Notably, COP1 expression is found to be SHH-dependent. COP1 targets p53 for degradation and thus regulates p53-responsive functions.

The history of BCG immunotherapy dates as early as 1976 when Morales and colleagues found its potential to treat superficial bladder cancer [42]. Further, BCG went on to be the first FDA-approved immunotherapy [43]. For a few decades now, BCG immunotherapy is either a standard treatment or constitutes the treatment regime for several cancers. Though the success of such therapies has been tremendous, a significant set of the patients fail BCG therapy. In case of bladder cancer, while $20-30 \%$ of patients fail the therapy, $30-50 \%$ of them exhibit tumor recurrence [27]. Such cases of failed adjuvant or neoadjuvant BCG therapies and tumor recurrence during various human cancer treatment regimes have initiated alternative or complementary therapies [44]. While several mechanisms of action of BCG immunotherapies have been proposed and tested, we lack clear understanding on the reasons for failed therapies [26]. In the present investigation, we found that the ability of BCG to inhibit apoptosis of cancer cells could be one of the probable reasons that hinder tumor clearance during 


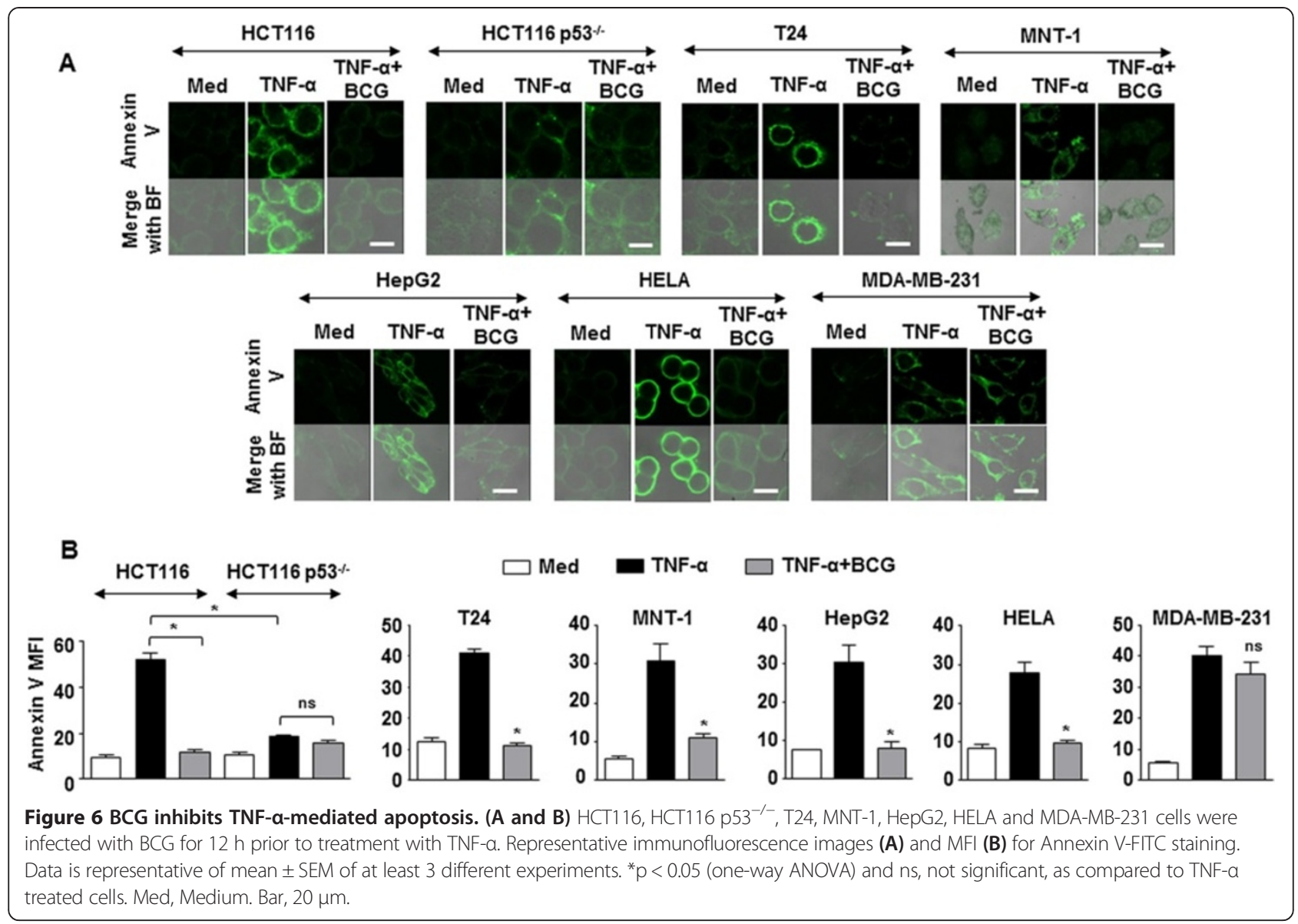

immunotherapy. Though BCG induces robust inflammatory responses during infection, there are multiple evidences of anti-inflammatory Th2 and regulatory $\mathrm{T}$ cell (Treg) functions stimulated by BCG $[45,46]$. Interestingly, both Th2 and Treg cells exhibit pro-tumor effects in cancers $[47,48]$. Evasion of protective immune responses by BCG could also contribute to failed immunotherapy.

Application of TNF- $\alpha$ as potent cytokine for cancer biotherapy is due to its ability to mediate functions such as apoptosis. To overcome the systemic toxicity associated with such treatments, various strategies including cell-based therapies and gene therapies are under study which aims at site-specific expression of TNF- $\alpha$ [44]. Our study indicates that BCG downregulates TNF- $\alpha$ arbitrated apoptosis in the tested human cancer cell lines, A549, HCT116, T24, MNT-1, HepG2 and HELA. Interestingly, results in MDA-MB-231 human breast adenocarcinoma cells and HCT116 $\mathrm{p} 53^{-/-}$human colorectal carcinoma cells were the outliers. While absence of p53 can be attributed to the reduced TNF- $\alpha$ responses and diminished p53 functions in HCT116 $\mathrm{p} 53^{-/-}$cells, the possible reasons for the observations in
MDA-MB-231 with mutant p53 is currently under investigation. Further, though BCG treatment, by itself, can induce the expression of TNF- $\alpha$ in immune cells [23] and during BCG therapy [49], in the current study we addressed the ability of BCG to revert the TNF- $\alpha$ responses such as apoptosis during therapy. However, BCG failed to protect A549 cells against apoptosis induced by other death ligands of the TNF family such as TRAIL (Additional file 2: Figure S2). This observation is in line with the available literature that suggests that TRAIL induces apoptosis regardless of the p53 status in the cells [50]. Further, the specificity of the response was also assessed in macrophages, the main host of Mycobacterium. BCG infection of PMA-stimulated THP-1 macrophages failed to protect the cells from TNF- $\alpha$-mediated apoptosis (Additional file 3: Figure S3). This observation is in line with existing literatures that suggest that BCG induces apoptosis of the infected macrophages [51-53]. However, TNF- $\alpha$-induced $p 53$ expression was slightly downregulated during BCG infection of THP-1 cells. This suggests that there could be p53-independent mechanisms that BCG adopts for inducing apoptosis in macrophages [53]. Interestingly, treatment of A549 cells with BCG lysate, 
Pam3CSK4 (a TLR2/1 agonist), LPS (a TLR4 agonist) or R848 (a TLR7/8 agonist) showed induced COP1 expression and corresponding inhibition of TNF- $\alpha$-mediated p53 expression (Additional file 4: Figure S4A). However, except for LPS treatment, induction of apoptosis by TNF- $\alpha$ was downregulated by BCG lysate, Pam3CSK4 and R848 (Additional file 4: Figure $S 4 B$ and $C$ ). This suggests that stimulation of TLR $1 / 2 / 7 / 8$ could lead to suppression of TNF- $\alpha$-mediated responses in A549 cells.

\section{Conclusion}

In summary, we show that BCG suppressed the apoptotic functions of TNF- $\alpha$ in many cancer cell lines thereby promoting tumorigenesis. Here, $\mathrm{SHH}$-responsive E3 ligase COP1 aids in downregulating the TNF- $\alpha$-driven p53 expression and functions (Figure 7). Our results suggest that inhibition of apoptosis by $M$. bovis BCG could result in unsuccessful intravesical immunotherapy. Thus the study underscores the need for alternate agents for immunotherapy.

\section{Methods}

\section{Cell-lines and bacteria}

A549 human lung adenocarcinoma, HCT116, HCT116 $\mathrm{p} 3^{-/-}$human colorectal carcinoma, HELA human cervical adenocarcinoma cell lines and THP-1 human monocytic cells were cultured in DMEM (Gibco-Life Technologies, Carlsbad, CA, USA) containing 10\% FBS
(Gibco-Life Technologies). T24 human urinary bladder carcinoma cells were cultured in McCoy's 5A modified medium (Sigma-Aldrich, St. Louis, MO, USA). MNT-1 human melanoma cell line was maintained in MNT-1 media containing DMEM supplemented with $10 \%$ AIMV medium, 15\% FBS, 1\% glutamine, 1\% sodium pyruvate and $1 \%$ non-essential amino acids (all from Gibco-Life Technologies). HepG2 human hepatocellular carcinoma cell line was cultured in EMEM (Sigma-Aldrich) containing 10\% FBS. M. bovis BCG Pasteur 1173P2 was obtained from Pasteur Institute, Paris, France. BCG-RFP was generated in the lab. Bacteria were grown to mid-log phase and used at 10 multiplicity of infection (MOI) in all the experiments.

\section{Reagents and antibodies}

General laboratory chemicals were obtained from SigmaAldrich or Merck Millipore (Darmstadt, Germany). TNF- $\alpha$ and TRAIL were purchased from PeproTech Inc (Rocky Hill, NJ, USA). Pam3CSK4, LPS and R848 were purchased from ImmunoTools (Friesoythe, Germany). Anti$\beta$-ACTIN antibody was bought from Sigma-Aldrich. Anti-p53 and anti-COP1 antibodies were obtained from Santa Cruz Biotechnology Ltd (Dallas, Texas, USA). Anti-SHH, anti-GLI1, anti-PTCH1, anti-NUMB, anti-Ser9 phospho GSK-3 $\beta$ and anti-UbK48 were purchased from Cell Signaling Technology (Danvers, MA, USA). Annexin $\mathrm{V}$ - fluorescein isothiocyanate (FITC) was from Miltenyi

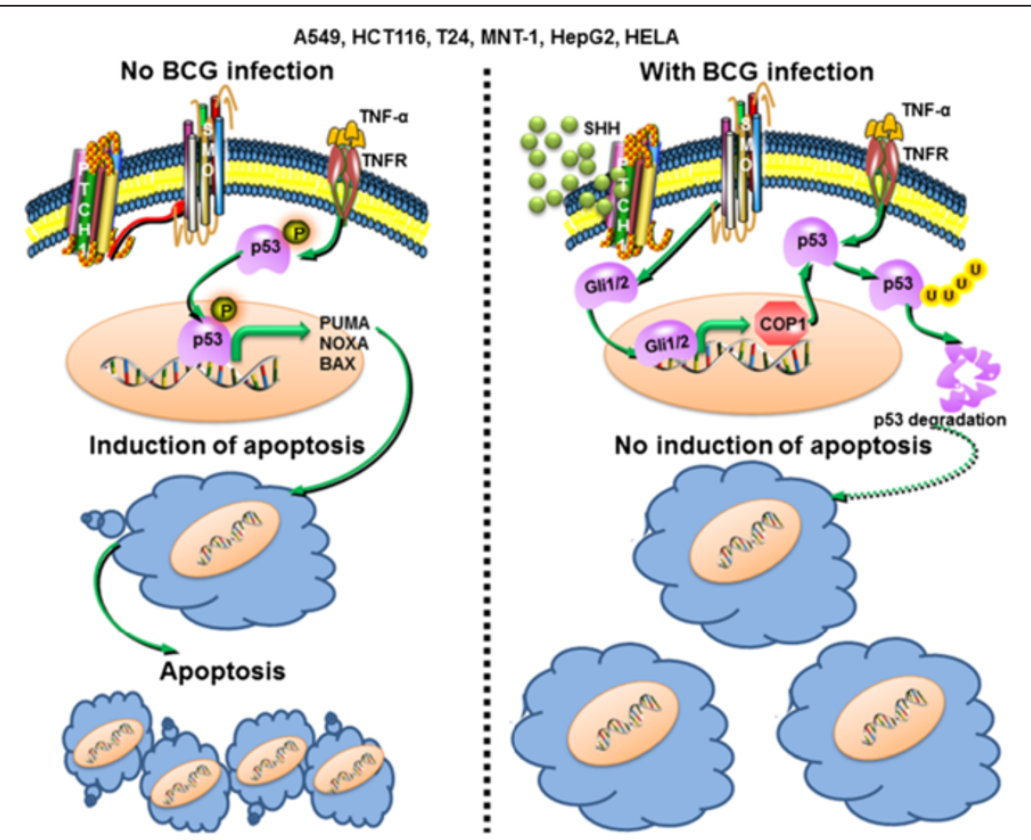

Figure 7 Model. TNF-a regulates the progression of cancers by promoting p53-dependent apoptosis. However, the presence of BCG induces SHH signaling-dependent expression of COP1, a regulator of p53 activity. COP1 targets p53 for ubiquitin-mediated proteasomal degradation. These events culminate into inhibition of apoptosis and promotion of tumorigenicity. 
Biotech (Bergisch Gladbach, Germany). HRP conjugated anti-rabbit IgG and anti-mouse IgG were obtained from Jackson ImmunoResearch (West Grove, PA, USA).

\section{Xenografts in nude mice}

Nude mice (nu/nu) were maintained in the Central Animal Facility, Indian Institute of Science. All studies involving mice were carried out after the approval from the Institutional Ethics Committee for Animal Experimentation as well as from Institutional Biosafety Committee. Subcutaneous injection of mice with $4 \times 10^{6}$ A549 cells into its right flank was carried out. The xenografts were monitored regularly for a period of 30 days and tumor sizes were measured with a caliper. After 30 days, tumors were excised and the tumor volume was measured by the formulae $\mathrm{V}=\left(\mathrm{LxW}^{2}\right) \times 0.52$, where $\mathrm{V}$ is volume, $\mathrm{L}$ is length, $\mathrm{W}$ is width.

\section{Treatment with pharmacological reagents}

In the experiments utilizing inhibitors, cells were treated with the given inhibitor for $1 \mathrm{~h}$ before the experimental treatments at following concentrations: Cyclopamine $(10 \mu \mathrm{M})$, Betulinic Acid $(10 \mu \mathrm{M})$ (both from CalbiochemMerck Millipore, Darmstadt, Germany). DMSO at $0.1 \%$ concentration was used as the vehicle control. In all experiments involving pharmacological reagents, a tested concentration was used after careful titration experiments assessing the viability of the cells using the MTT (3-(4,5-Dimethylthiazol-2-yl)-2,5-diphenyltetrazolium bromide) assay.

\section{MTT assay}

Survival of the A549 cells were evaluated by MTT assay as described previously [54]. Briefly, a cell density of 1.5 $\times 10^{3} /$ well was seeded in a 96-well plate and the experiment was carried out. $20 \mu \mathrm{l}$ of $5 \mathrm{mg} / \mathrm{ml} \mathrm{MTT} \mathrm{reagent}$ was added to the wells $3 \mathrm{~h}$ prior to the completion of the experiment. The formazan crystals formed in the living cells were dissolved in $200 \mu \mathrm{l}$ of DMSO which was added to the washed cells. Absorbance of the solution was measured at $550 \mathrm{~nm}$ using an ELISA reader (Molecular Devices, Sunnyvale, CA, USA).

\section{Transfection studies}

A549 cells were transiently transfected with $5 \mu \mathrm{g}$ of $\mathrm{COP} 1, \mathrm{p} 53, \mathrm{SHH}$ or $\beta$-galactosidase overexpression constructs or COP1, p53 luciferase constructs using low m.w. polyethylenimine (Sigma-Aldrich). In case of experiments involving siRNA, A549 cells were transfected with 100 nM siRNA. SHH, GLI1, COP1, non-targeting siRNA and siGLO Lamin A/C were obtained from Dharmacon, Inc. (Lafayette, CO, USA) as siGENOME ${ }^{\mathrm{TM}}$ SMARTpool reagents, which contain a pool of four different doublestranded RNA oligonucleotides. Transfection efficiency was found to be more than $70 \%$ in all the experiments as determined by counting the number of siGLO Lamin A/C positive cells. In all cases, $48 \mathrm{~h}$ post-transfection, the cells were treated as indicated and processed for analysis.

\section{Immunofluorescence}

Cells were seeded on to coverslips and treated as indicated. Annexin V-FITC staining was carried out according to manufacturer's instructions. The cells were fixed with $3.7 \%$ paraformaldehyde for $15 \mathrm{~min}$ at room temperature and the coverslips were mounted on a slide with glycerol. Confocal images were taken on Zeiss LSM 710 and Zeiss LSM 510 Meta confocal laser scanning microscopes (Carl Zeiss AG, Oberkochen, Germany) using a planApochromat 63X/1.4 Oil DIC objective and images were analyzed using ZEN 2009 software (Carl Zeiss AG).

\section{RNA isolation and quantitative real time RT-PCR}

Total RNA from experimentally treated cell lines was isolated using TRI reagent (Sigma-Aldrich). $2 \mu \mathrm{g}$ of total RNA was converted into cDNA using First strand cDNA synthesis kit (Bioline, London, UK). Quantitative real time RT-PCR for quantification of the target gene expression was performed using SYBR Green PCR mixture (KAPA Biosystems, Woburn, MA, USA). All the experiments were repeated at least three times independently to ensure the reproducibility of the results. GAPDH was used as internal control. Additional file 5: Table S1 contains the primers used for quantitative real time RT-PCR.

\section{Immunoblotting}

RIPA buffer [50 mM Tris-HCl (pH 7.4), 1\% NP-40, 0.25\% Sodium deoxycholate, $150 \mathrm{mM} \mathrm{NaCl}, 1 \mathrm{mM}$ EDTA, $1 \mathrm{mM}$ PMSF, $1 \mu \mathrm{g} / \mathrm{ml}$ of each aprotinin, leupeptin, pepstatin, $1 \mathrm{mM} \mathrm{Na}_{3} \mathrm{VO}_{4}$ and $1 \mathrm{mM} \mathrm{NaF}$ ] was used to obtain the total cell lysates. Protein in each sample was estimated using Bradford assay; equal amount of protein was resolved on a $12 \%$ SDS-polyacrylamide gel and transferred to polyvinylidene difluoride (PVDF) membranes (Millipore) by the semi-dry transfer (Bio-Rad, Hercules, CA, USA) method. Blocking with 5\% nonfat dry milk powder in TBST [20 mM Tris- $\mathrm{HCl}$ (pH 7.4), $137 \mathrm{mM} \mathrm{NaCl}$, and $0.1 \%$ Tween 20] for $60 \mathrm{~min}$ was done to prevent nonspecific binding. The blots were incubated overnight at $4^{\circ} \mathrm{C}$ with primary antibody followed by incubation with antirabbit-HRP or anti-mouse-HRP secondary antibody in 5\% BSA for $2 \mathrm{~h}$. The immunoblots were developed with enhanced chemiluminescence detection system (PerkinElmer, Waltham, MA, USA) as per manufacturer's instructions. $\beta$-ACTIN was used as loading control. Blots were incubated in the stripping buffer [62.5 mM Tris- $\mathrm{HCl}(\mathrm{pH} 6.8)$, $2 \%$ SDS and $0.7 \% \beta$-mercaptoethanol] at $60^{\circ} \mathrm{C}$ on a shaker for probing another protein in the same region of the PVDF membrane. 


\section{Luciferase assays}

Transfected cells were lysed in Reporter lysis buffer (Promega, Madison, WI, USA) and assayed for luciferase activity using Luciferase Assay Reagent (Promega) as per the manufacturer's instructions. $\beta$-galactosidase activity utilizing $\mathrm{O}$-nitrophenol $\beta$-D-galactopyranoside (HiMedia Laboratories Pvt Ltd, Mumbai, Maharashtra, India) was performed to normalize the transfection efficiencies.

\section{Chromatin immunoprecipitation assay}

Chromatin immunoprecipitation (ChIP) assay was carried out using a protocol provided by Upstate Biotechnology, Inc. (Lake Placid, NY, USA), with certain modifications. Briefly, cells were fixed with $1.42 \%$ formaldehyde for $15 \mathrm{~min}$ at room temperature followed by inactivation of formaldehyde using $125 \mathrm{mM}$ glycine. Modified RIPA buffer containing 1\% Triton X-100 was used for cell lysis and samples were sonicated to obtain sheared chromatin fragments of $500 \mathrm{bp}$. Anti-GLI1 or rabbit preimmune sera immunoprecipitated DNA was purified and analyzed by quantitative PCR. All results were normalized to amplification of $28 \mathrm{~S}$ rRNA. ChIP experiments were repeated at least three times and the primers utilized are listed in Additional file 5: Table S1.

\section{Immunoprecipitation assay}

Immunoprecipitation (IP) assays were carried out using protocol provided by Millipore with certain modifications. Briefly, the cells were gently lysed in ice-cold RIPA buffer on an orbital shaker. The cell lysates were incubated with anti-UbK48, anti-p53, anti-COP1 or rabbit preimmune sera at $4^{\circ} \mathrm{C}$ for $2 \mathrm{~h}$ on an orbital shaker. The immunocomplexes were captured using Protein A (Bangalore Genei) agarose at $4^{\circ} \mathrm{C}$ for $2 \mathrm{~h}$. The beads were harvested, washed, and boiled in $5 \times$ Laemmli buffer for $10 \mathrm{~min}$. The samples were separated by SDS-PAGE and further subjected for immunoblotting.

\section{Statistical analysis}

Levels of significance for comparison between samples were determined by the Student $t$ test distribution and one-way ANOVA. The data in the graphs are expressed as the mean \pm SEM for 5 or 6 values from 3 independent experiments and p values $<0.05$ were defined as significant. GraphPad Prism 5.0 software (GraphPad Software, Inc., San Diego, CA, USA) was used for all the statistical analysis.

\section{Additional files}

Additional file 1: Figure S1. BCG inhibits TNF-a-induced apoptosis. (A) A549 cells were infected with BCG-RFP for $12 \mathrm{~h}$ prior to TNF- $\mathrm{a}$ treatment. Representative immunofluorescence images for Annexin
V-FITC staining and BCG-RFP. Data is representative of 3 different experiments. Med, Medium. Bar, $5 \mu \mathrm{m}$.

Additional file 2: Figure S2. BCG failed to downregulate TRAIL-induced apoptosis. (A) A549 cells were infected with BCG for $12 \mathrm{~h}$ prior to treatment with $20 \mathrm{ng} / \mathrm{ml}$ TRAIL. Representative immunofluorescence images and MFI for Annexin V-FITC staining. Data is representative of mean \pm SEM of at least 3 different experiments. ns, not significant, as compared to TNF-a treated cells. Med, Medium. Bar, $20 \mu \mathrm{m}$.

Additional file 3: Figure S3. BCG failed to downregulate TNF-a-induced apoptosis in macophages. (A and B) PMA-stimulated THP-1 cells were infected with BCG for $12 \mathrm{~h}$ prior to TNF-a treatment. Expression of p53 and COP1 were assessed by immunoblotting with total cell lysate (A) and representative immunofluorescence images and MFI for Annexin V-FITC staining (B). Data is representative of mean \pm SEM of at least 3 different experiments and all blots are representative of 3 independent experiments. ns, not significant, as compared to TNF-a treated cells. Med, Medium. Bar, $20 \mu \mathrm{m}$.

Additional file 4: Figure S4. Ability of various innate receptor agonists to inhibit TNF-a-induced apoptosis. (A-C) A549 cells were either infected with BCG or stimulated with BCG lysate, Pam3CSK4 $(1 \mu \mathrm{g} / \mathrm{ml})$, LPS (50 ng/ml) or R848 $(1 \mu \mathrm{g} / \mathrm{ml})$ for $12 \mathrm{~h}$ prior to treatment with TNF-a. Immunoblotting analysis of $\mathrm{p} 53$ and COP1 (A) and MFI (B) and representative immunofluorescence images (C) for Annexin V-FITC staining. Data is representative of mean \pm SEM of at least 3 different experiments and all blots are representative of 3 independent experiments. ${ }^{*} p<0.05$ (one-way ANOVA) and ns, not significant, as compared to TNF-a treated cells. Med, Medium. Bar, $20 \mu \mathrm{m}$.

Additional file 5: Table S1. Primers used in the study.

\section{Abbreviations}

BCG: M. bovis Bacillus Calmette-Guérin; ChIP: Chromatin immunoprecipitation; COP1: Constitutively photomorphogenic 1; IP: Immunoprecipitation; MTT: 3-(4,5-Dimethylthiazol-2-yl)-2,5-diphenyltetrazolium bromide; PTCH1: Patched-1; SMO: Smoothened; SHH: Sonic hedgehog; Treg: Regulatory T cell; TNF: Tumor necrosis factor.

\section{Competing interests}

The authors declare that they have no competing interests.

\section{Authors' contributions}

SH designed, performed, analyzed data and wrote the paper. DSG and KB designed, performed and analyzed data. VS performed and analyzed data. KNB conceived the study and participated in its design, analysis and helped to draft the manuscript. All authors read and approved the final manuscript.

\section{Acknowledgements}

We thank the Central Animal facility, Indian Institute of Science (IISC) for providing mice for experimentation. We sincerely thank Dr. Andrew McMahon, Harvard University, Massachusetts, USA for kind gift of $\mathrm{SHH}$ overexpression construct. We thank Dr. Kumaravel Somasundaram, IISc for p53 expression and luciferase constructs and human cell lines, Dr. Saumitra Das (IISc), Dr. G. Subba Rao (IISC), Dr. Annapoorni Rangarajan (IISc), Dr. Jagadeesh Bayry (INSERM, Paris, France), Dr. Clotilde Théry and Dr. Isabelle Bernard-Pierrot (both from Institut Curie, Paris, France) for providing various human cell lines. We thank Dr. M. S. Patole, National Center for Cell Sciences (NCCS), Pune, India and the repository at NCCS for providing T24 human urinary bladder carcinoma cells. Samrajyam Nara and Pannaga of the MCB imaging facility and Deepti Bapat of IISC confocal facility are acknowledged for their help. We acknowledge Shambhuprasad T. K for help during the investigation.

This study is supported by funds from the Department of Biotechnology (DBT), Department of Science and Technology (DST), Council for Scientific and Industrial Research (CSIR), Indian Council of Medical Research (ICMR) and the Indo-French Center for Promotion of Advanced Research (IFCPAR/CEFIPRA). Infrastructure support from ICMR (Center for advanced study in Molecular Medicine), DST (FIST) and UGC (special assistance) (KNB); fellowships from IISC (SH and KB) and CSIR (DSG and VS) are acknowledged. 
Received: 30 March 2014 Accepted: 5 September 2014

Published: 11 September 2014

\section{References}

1. Bermudez LE, Goodman J: Mycobacterium tuberculosis invades and replicates within type II alveolar cells. Infect Immun 1996, 64:1400-1406.

2. Li Y, Wang Y, Liu X: The role of airway epithelial cells in response to mycobacteria infection. Clin Dev Immunol 2012, 2012:791392.

3. Lin $Y$, Zhang M, Barnes PF: Chemokine production by a human alveolar epithelial cell line in response to Mycobacterium tuberculosis. Infect Immun 1998, 66:1121-1126.

4. Flynn JL, Chan J: Immunology of tuberculosis. Annu Rev Immunol 2001, 19:93-129.

5. Cooper AM: Cell-mediated immune responses in tuberculosis. Annu Rev Immunol 2009, 27:393-422.

6. Eggermont AM, de Wilt JH, ten Hagen TL: Current uses of isolated limb perfusion in the clinic and a model system for new strategies. Lancet Oncol 2003, 4:429-437.

7. Grunhagen DJ, de Wilt JH, Graveland WJ, van Geel AN, Eggermont AM: The palliative value of tumor necrosis factor alpha-based isolated limb perfusion in patients with metastatic sarcoma and melanoma. Cancer 2006, 106:156-162

8. Yu YH, Liao CC, Hsu WH, Chen HJ, Liao WC, Muo CH, Sung FC, Chen CY: Increased lung cancer risk among patients with pulmonary tuberculosis: a population cohort study. J Thorac Oncol 2011, 6:32-37.

9. Liang HY, Li XL, Yu XS, Guan P, Yin ZH, He QC, Zhou BS: Facts and fiction of the relationship between preexisting tuberculosis and lung cancer risk: a systematic review. Int J Cancer 2009, 125:2936-2944

10. Dheda K, Booth H, Huggett JF, Johnson MA, Zumla A, Rook GA: Lung remodeling in pulmonary tuberculosis. J Infect Dis 2005, 192:1201-1209.

11. Coussens LM, Werb Z: Inflammation and cancer. Nature 2002, 420:860-867.

12. Fridman JS, Lowe SW: Control of apoptosis by p53. Oncogene 2003, 22:9030-9040.

13. Lowe SW, Cepero E, Evan G: Intrinsic tumour suppression. Nature 2004 432:307-315.

14. de Stanchina E, Lowe SW: Tumour suppression: something for nothing? Nat Cell Biol 2002, 4:E275-E276.

15. Dornan D, Wertz I, Shimizu H, Arnott D, Frantz GD, Dowd P, O'Rourke K, Koeppen $\mathrm{H}$, Dixit VM: The ubiquitin ligase COP1 is a critical negative regulator of p53. Nature 2004, 429:86-92.

16. Wu H, Lozano G: NF-kappa B activation of p53. A potential mechanism for suppressing cell growth in response to stress. J Biol Chem 1994, 269:20067-20074

17. Varjosalo M, Taipale J: Hedgehog: functions and mechanisms. Genes Dev 2008, 22:2454-2472.

18. Merchant AA, Matsui W: Targeting Hedgehog-a cancer stem cell pathway. Clin Cancer Res 2010, 16:3130-3140.

19. Ingham PW, McMahon AP: Hedgehog signaling in animal development: paradigms and principles. Genes Dev 2001, 15:3059-3087.

20. Goh AM, Coffill CR, Lane DP: The role of mutant p53 in human cancer. J Pathol 2011, 223:116-126.

21. van Horssen R, Ten Hagen TL, Eggermont AM: TNF-alpha in cancer treatment: molecular insights, antitumor effects, and clinical utility. Oncologist 2006, 11:397-408.

22. Abe Y, Oda-Sato E, Tobiume K, Kawauchi K, Taya Y, Okamoto K, Oren M, Tanaka N: Hedgehog signaling overrides p53-mediated tumor suppression by activating Mdm2. Proc Natl Acad Sci U S A 2008, 105:4838-4843

23. Ghorpade DS, Holla S, Kaveri SV, Bayry J, Patil SA, Balaji KN: Sonic hedgehog-dependent induction of microRNA 31 and microRNA 150 regulates Mycobacterium bovis BCG-driven toll-like receptor 2 signaling. Mol Cell Biol 2013, 33:543-556.

24. Holla S, Kurowska-Stolarska M, Bayry J, Balaji KN: Selective inhibition of IFNG-induced autophagy by Mir155- and Mir31-responsive WNT5A and SHH signaling. Autophagy 2014, 10:311-330.

25. Mitropoulos DN: Novel insights into the mechanism of action of intravesical immunomodulators. In Vivo 2005, 19:611-621.

26. Yates DR, Brausi MA, Catto JW, Dalbagni G, Roupret M, Shariat SF, Sylvester RJ, Witjes JA, Zlotta AR, Palou-Redorta J: Treatment options available for bacillus Calmette-Guerin failure in non-muscle-invasive bladder cancer. Eur Urol 2012, 62:1088-1096.
27. Zaharoff DA, Hoffman BS, Hooper HB, Benjamin CJ Jr, Khurana KK, Hance KW, Rogers CJ, Pinto PA, Schlom J, Greiner JW: Intravesical immunotherapy of superficial bladder cancer with chitosan/interleukin-12. Cancer Res 2009, 69:6192-6199.

28. Hirschowitz EA, Yannelli JR: Immunotherapy for lung cancer. Proc Am Thorac Soc 2009, 6:224-232

29. Murala S, Alli V, Kreisel D, Gelman AE, Krupnick AS: Current status of immunotherapy for the treatment of lung cancer. J Thorac Dis 2010 2:237-244.

30. Mosolits S, Nilsson B, Mellstedt H: Towards therapeutic vaccines for colorectal carcinoma: a review of clinical trials. Expert Rev Vaccines 2005, 4:329-350.

31. Gutterman JU, Mavligit G, McBride C, Frei E III, Freireich EJ, Hersh EM: Active immunotherapy with B.C.G. for recurrent malignant melanoma. Lancet 1973, 1:1208-1212

32. Stewart JH, Levine EA: Role of bacillus Calmette-Guerin in the treatment of advanced melanoma. Expert Rev Anticancer Ther 2011, 11:1671-1676.

33. Butterfield LH: Recent advances in immunotherapy for hepatocellular cancer. Swiss Med Wkly 2007, 137:83-90.

34. Lu X, Wu L, Liu Z, Xie L, Wang S: Peripheral blood mononuclear cells inhibit proliferation and promote apoptosis of HeLa cells following stimulation with Bacillus Calmette-Guerin. Exp Ther Med 2013, 5:561-566.

35. Sato K, Tomioka H, Shimizu T, Gonda T, Ota F, Sano C: Type II alveolar cells play roles in macrophage-mediated host innate resistance to pulmonary mycobacterial infections by producing proinflammatory cytokines. J Infect Dis 2002, 185:1139-1147.

36. Lane DP: Cancer. p53, guardian of the genome. Nature 1992, 358:15-16.

37. Lowe SW, Ruley HE, Jacks T, Housman DE: p53-dependent apoptosis modulates the cytotoxicity of anticancer agents. Cell 1993, 74:957-967.

38. Marine JC: Spotlight on the role of COP1 in tumorigenesis. Nat Rev Cancer 2012, 12:455-464

39. Adjei $\mathrm{AA}$, Hidalgo M: Intracellular signal transduction pathway proteins as targets for cancer therapy. J Clin Oncol 2005, 23:5386-5403.

40. Narayana $Y$, Balaji KN: NOTCH1 up-regulation and signaling involved in Mycobacterium bovis BCG-induced SOCS3 expression in macrophages. J Biol Chem 2008, 283:12501-12511.

41. Bansal K, Trinath J, Chakravortty D, Patil SA, Balaji KN: Pathogen-specific TLR2 protein activation programs macrophages to induce Wnt-betacatenin signaling. J Bio/ Chem 2011, 286:37032-37044

42. Morales A, Eidinger D, Bruce AW: Intracavitary Bacillus Calmette-Guerin in the treatment of superficial bladder tumors. J Urol 1976, 116:180-183.

43. Grossman HB, O'Donnell MA, Cookson MS, Greenberg RE, Keane TE: Bacillus calmette-guerin failures and beyond: contemporary management of non-muscle-invasive bladder cancer. Rev Urol 2008, 10:281-289.

44. Cai W, Kerner ZJ, Hong H, Sun J: Targeted cancer therapy with tumor necrosis factor-alpha. Biochem Insights 2008, 2008:15-21.

45. Martino A, Sacchi A, Sanarico N, Spadaro F, Ramoni C, Ciaramella A, Pucillo LP Colizzi V, Vendetti S: Dendritic cells derived from BCG-infected precursors induce Th2-like immune response. J Leukoc Biol 2004, 76:827-834.

46. Scott-Browne JP, Shafiani S, Tucker-Heard G, Ishida-Tsubota K, Fontenot JD, Rudensky AY, Bevan MJ, Urdahl KB: Expansion and function of Foxp3expressing T regulatory cells during tuberculosis. J Exp Med 2007, 204:2159-2169.

47. Mougiakakos D, Choudhury A, Lladser A, Kiessling R, Johansson CC: Regulatory T cells in cancer. Adv Cancer Res 2010, 107:57-117.

48. Protti MP, De Monte L: Cross-talk within the tumor microenvironment mediates Th2-type inflammation in pancreatic cancer. Oncoimmunology 2012, 1:89-91

49. Shin JS, Park JH, Kim JD, Lee JM, Kim SJ: Induction of tumour necrosis factor-alpha (TNF-alpha) mRNA in bladders and spleens of mice after intravesical administration of bacillus Calmette-Guerin. Clin Exp Immunol 1995, 100:26-31.

50. Wang S, El-Deiry WS: TRAIL and apoptosis induction by TNF-family death receptors. Oncogene 2003, 22:8628-8633.

51. Riendeau CJ, Kornfeld H: THP-1 cell apoptosis in response to Mycobacterial infection. Infect Immun 2003, 71:254-259.

52. Lee J, Remold HG, leong MH, Kornfeld H: Macrophage apoptosis in response to high intracellular burden of Mycobacterium tuberculosis is mediated by a novel caspase-independent pathway. J Immunol 2006, 176:4267-4274. 
53. Ghorpade DS, Leyland R, Kurowska-Stolarska M, Patil SA, Balaji KN: MicroRNA-155 is required for Mycobacterium bovis BCG-mediated apoptosis of macrophages. Mol Cell Biol 2012, 32:2239-2253.

54. Wajapeyee N, Somasundaram K: Cell cycle arrest and apoptosis induction by activator protein 2alpha (AP-2alpha) and the role of p53 and p21WAF1/CIP1 in AP-2alpha-mediated growth inhibition. J Biol Chem 2003, 278:52093-52101.

doi:10.1186/1476-4598-13-210

Cite this article as: Holla et al:: Mycobacterium bovis BCG promotes

tumor cell survival from tumor necrosis factor-a-induced apoptosis. Molecular Cancer 2014 13:210

\section{Submit your next manuscript to BioMed Central and take full advantage of:}

- Convenient online submission

- Thorough peer review

- No space constraints or color figure charges

- Immediate publication on acceptance

- Inclusion in PubMed, CAS, Scopus and Google Scholar

- Research which is freely available for redistribution 